\title{
RESEARCH
}

Open Access

\section{Wild edible ectomycorrhizal fungi: an underutilized food resource from the rainforests of Tshopo province (Democratic Republic of the Congo)}

Héritier Milenge Kamalebo ${ }^{1,2^{*}}$ (1) and André De Kesel ${ }^{3}$

\begin{abstract}
Background: Ectomycorrhizal (EcM) fungi constitute a source of income as well as proper food with considerable nutritional value. Although edible EcM fungi are highly diverse and expected to host considerable nutritional attributes, only few studies focus on their use and promotion in the province of Tshopo (DR Congo). This study provides original ethnomycological and diversity data on edible ectomycorrhizal rainforest fungi from the Man-andBiosphere reserve of Yangambi and the reserve of Yoko.

Methods: The list of edible fungi follows the current taxonomy. Taxa were collected in plots situated in different types of rainforests. Each taxon is supported by herbarium reference specimens. Ethnomycological data on locally consumed EcM fungi were collected from randomly selected people living near the Man-and-Biosphere reserve of Yangambi and the Yoko reserve. People were interviewed using a semi-structured questionnaire. The interview campaign involved 160 informants, all randomly selected from 6 different ethnic communities.

Results: The results reveal that rainforests from the Yangambi Biosphere reserve and Yoko forest reserve provide a relatively high number of edible fungi, more than local people actually use. Mixed forest stands hold the highest diversity in saprotrophic edible fungi ( $p$ value $<0.001)$ while no significant difference ( $p$ value $>0.05$ ) was observed in the number of saprotrophic and EcM fungi within monodominant forests. In spite of being accessible, this renewable natural resource is underexploited. Although a wide array of EcM fungi is available in primary forests dominated by ectomycorrhizal trees, local people's major interest goes to the saprotrophic fungi from areas with degraded mixed forests.

Conclusion: The lack of local interest for EcM fungi is probably related to the considerable distance people have to cover to collect them. As a result, the edible EcM fungi from the Tshopo area represent a potentially interesting but underutilized resource.
\end{abstract}

Keywords: Edible ectomycorrhizal fungi, Fungal consumption, Tshopo, Democratic Republic of the Congo

\footnotetext{
* Correspondence: kamaleboheritier@gmail.com

${ }^{1}$ Faculté des Sciences, Université de Kisangani, BP 2012, Kisangani,

Democratic Republic of the Congo

${ }^{2}$ Centre de Recherches Universitaires du Kivu (CERUKI-ISP), BP 854, Bukavu,

Democratic Republic of the Congo

Full list of author information is available at the end of the article
}

(c) The Author(s). 2020 Open Access This article is distributed under the terms of the Creative Commons Attribution 4.0 International License (http://creativecommons.org/licenses/by/4.0/), which permits unrestricted use, distribution, and reproduction in any medium, provided you give appropriate credit to the original author(s) and the source, provide a link to the Creative Commons license, and indicate if changes were made. The Creative Commons Public Domain Dedication waiver (http://creativecommons.org/publicdomain/zero/1.0/) applies to the data made available in this article, unless otherwise stated. 


\section{Introduction}

In many regions of the world, including tropical Africa, edible fungi often constitute a source of income and food, with a non-negligible nutritional value [1-6]. According to De Kesel et al. [4] and Degreef et al. [7], documenting the relative importance and potential of locally used EcM fungi remains vital for improving strategies for their conservation and promoting sustainable use. Edible EcM fungi provide high-quality and important amount of crude protein, minerals and carbohydrates, fats, etc. $[2,3,5,6]$. The amount of fungal crude protein for instance is generally ranked between 19 and 35\% of the dry mass [2]. Furthermore, many species of edible EcM fungi provide higher amount of unsaturated fatty acids than saturated ones $[2,5]$. Unlike saturated fatty acids (found in high amount in animal fats), unsaturated fatty acids are suggested to be essential in the human diet [2]. The high proportion of unsaturated fatty acids and high percentage of linoleic acid found in edible EcM mushrooms lead to consider them as a healthy food [2]. In addition, the proteins of most of edible EcM taxa contain all nine essential amino acids (leucine, isoleucine, valine, tryptophan, lysine, threonine, phenylalanine, methionine, and histidine) used in humans' nutrition $[2,5]$.

EcM fungi constitute one of the most important groups of edible fungi worldwide. In tropical Africa, EcM fungi are massively consumed in the Zambezian region $[4,5,8-14]$, and in the savanna woodlands and open forest areas of the Soudanian region [15-17]. Within the Zambezian geographical zone, local people collect, sell, and consume impressive quantities of EcM taxa. The socio-economic interest for EcM fungi is considered a major incentive for obtaining strong and broadly supported forest conservation programs [4].

Within the rainforests of Tshopo province (Democratic Republic of the Congo), many species of edible EcM fungi are available. In this area, EcM fungi are typically found within primary forests dominated by EcM partner trees such as Gilbertiodendron dewevrei (De Wild.) J. Léonard, Brachystegia laurentii (De Wild.) Louis, Julbernardia seretii (De Wild.) Troupin, Uapaca guineensis Müll. Arg, and $U$. heudelotii Baillon [18, 19]. This study aims to assess the distribution and diversity of edible fungi within various types of rainforests found in the Man-and-Biosphere reserve of Yangambi and the Yoko reserve. We show how access and availability, as well as trophic level of fungi, affect the interest of local people, and how this in turn creates or annihilates opportunities for forest conservation.

\section{Materials and methods}

\section{Study site and sampling of fungi}

Fieldwork was done in the province of Tshopo, located $2^{\circ} \mathrm{N} 2^{\circ} \mathrm{S}$ and $22^{\circ} \mathrm{E} 28^{\circ} \mathrm{E}$ [20]. The vegetation from
Tshopo is mainly characterized by a tropical evergreen rainforest and some groves of semi-deciduous forests found on hills and plateaus [20-22]. These rainforests are mainly dominated by species such as Gilbertiodendron dewevrei (De Wild.) J. Léonard, Brachystegia laurentii (De Wild.) Louis, Scorodophloeus zenkeri Harms, Prioria balsamifera (Vermoesen) Breteler, and Julbernardia seretii (De Wild.) Troupin [20-24].

The fungi were collected between 2013 and 2016, mostly within plots situated in the rainforests of the Biosphere reserve of Yangambi $\left(0^{\circ} 51^{\prime} 01.62^{\prime \prime} \mathrm{N} ; 24^{\circ} 31^{\prime}\right.$ $43.53^{\prime \prime}$ E) and Yoko reserve $\left(0^{\circ} 17^{\prime} 34.9^{\prime \prime} \mathrm{N} ; 25^{\circ} 18^{\prime}\right.$ 27.4" E) (Fig. 1). Monitoring of plots follows Lodge et al. [25], and fungal sampling was performed within forests dominated by Gilbertiodendron dewevrei, Brachystegia laurentii, Julbernardia seretii, Uapaca heudelotii, and Uapaca guineensis and in mixed forests. Three plots of $100 \times 100 \mathrm{~m}$ were demarcated in each type of forest; each of them divided in a $20 \times 20 \mathrm{~m}$ grid. Due to the elongated shape of Uapaca heudelotii-dominated forests, more stretched plots were installed in this forest type. In each plot, the aboveground fruiting bodies of all EcM fungi were collected by walking parallel bands covering the entire plot [26]. Unidentifiable fruit bodies were photographed in situ and dried after notes were taken from their macromorphological features (following [18]). Voucher specimens were dried using a field drier [27] and deposited at the herbarium of Meise Botanic Garden (Belgium).

\section{Fungal identification}

The identification of voucher specimens was done using macroscopic and microscopic characteristics, as outlined in Eyi-Ndong et al. [18]. The available taxonomic literature only covers a fraction of the Central African rainforest fungi. The following contributions were used for identification: Heinemann [28], Heim [29], Pegler [30], Heinemann and Rammeloo [31-33], Buyck [8, 34, 35], De Kesel et al .[4, 9, 16], Verbeken and Walleyn [36], Eyi-Ndong et al. [18], as well as identification keys provided by the Fungus Flora of Tropical Africa (https://www.ffta-online.org/) and Edible Fungi of Tropical Africa (https://www.efta-online.org/). Species names and author's abbreviations largely follow Index Fungorum (http://www.indexfungorum.org/Names/Names.asp). Unidentified taxa were left out of the analysis.

\section{Ethnomycological data acquisition and treatment}

Ethnomycological data on locally consumed fungi were collected from local people living around the Man-andBiosphere reserve of Yangambi and the Yoko reserve. Data were collected using open, semi-structured interviews (paper fill-in questionnaires). The interviews involved mainly the head of the family, sometimes assisted by other family members. The questions focused basically on the informant's 


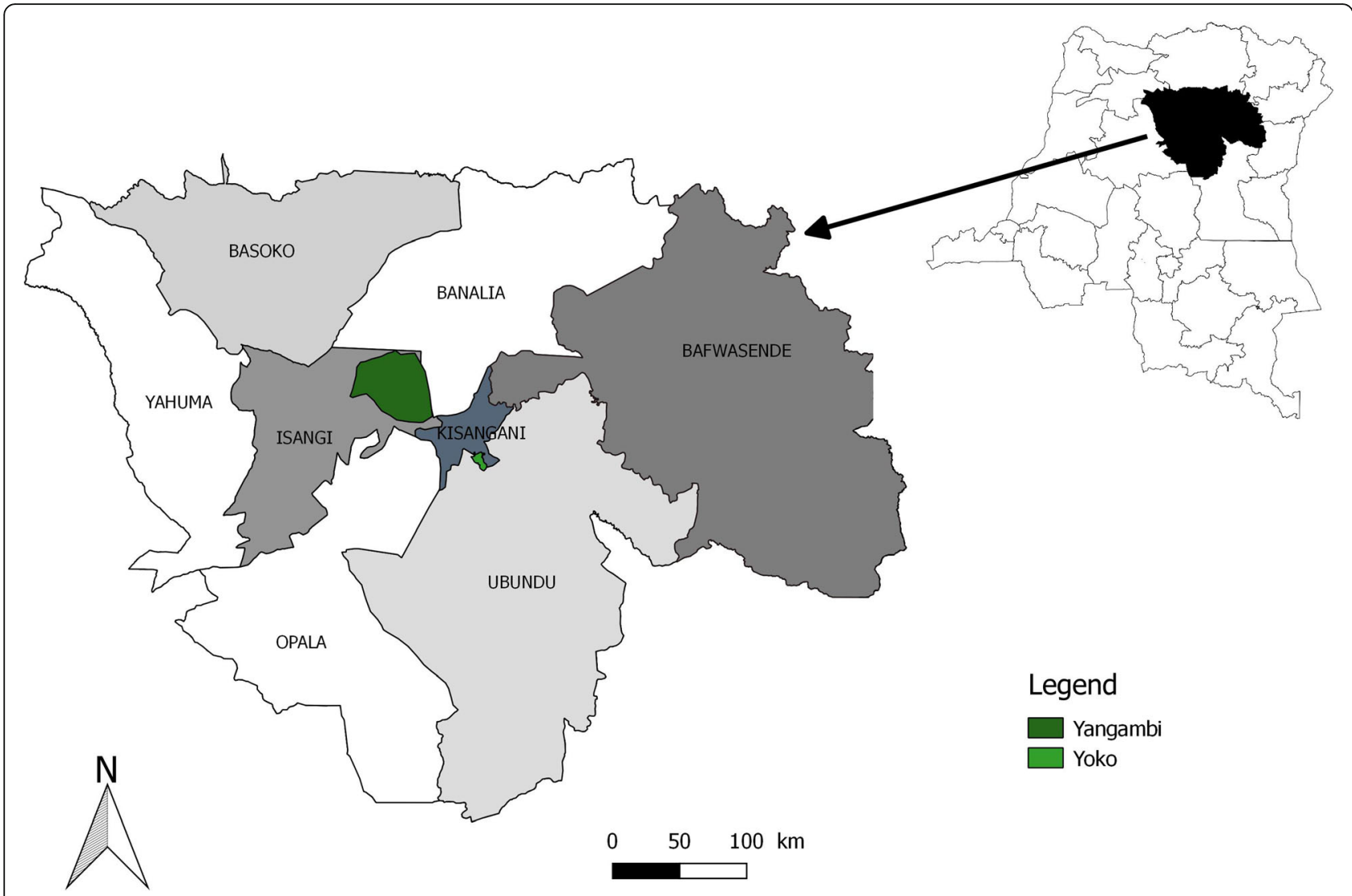

Fig. 1 Location of the study area

knowledge concerning the different locally consumed edible fungi. Interviews were obtained from 160 informants, all randomly selected, but belonging to one of 6 ethnic communities (Bakumu, Turumbu, Topoke, Lokele, Ngelema, and Ngando). The entire pool of informants counted 88 men (55\%) and 72 women (45\%), ranging from 16 to 72 years old. The interviewed communities live in four villages in the vicinity of the Man-and-Biosphere reserve of Yangambi (Yakako, Yalungu, Lyoli, and Lobiloto), 4 suburbs of Yangambi city (IFA, Lusambila, Ekuchu, and Manzikala), and 4 villages surrounding the Yoko forest reserve (Babogombe, Biaro, PK 48, and PK 25).

With an average of 600 households living in the studied area, the average number of studied households revolves around 25 per village (4.2\% of the entire pool). Within the 4 suburbs of Yangambi, the distribution of households was 150 (25\%), 120 (20\%), 80 (13.3\%), and $50(8.3 \%)$ respectively reported from Ekuchu, Lusambila, Manzikala, and IFA. According to Gumucio et al. [37], the sample size $(n)$ of the interviewed households should be calculated as follows: $n=$ $\frac{N}{1+N \times e^{2}}$, where $N$ is the total number of available households and $e$ is the level of precision. With a precision level of $\pm 7 \%$, the sample size was calculated by the following formula: $n=$
$\frac{600}{1+\left(N \times 0.07^{2}\right)} \approx 152$, households that were fitted to 160 informants. Referring to the mean distribution of households per sampling site (villages and suburbs of Yangambi), 7 households were interviewed from each of the 4 villages while 38 from Ekuchu, 30 from Lusambila, 20 from Manzikala, and 12 households from IFA. In each sampling site, all households were numbered. The first numbers referring to the considered sample size were selected randomly using the function "rand.between" of the Excel software.

The analyzed data only refer to locally eaten fungi and allow to present (per species) information on the edible mushrooms' cultural significance (EMCS). According to Pieroni [38], the edible mushrooms' cultural significance index refers to the importance or the role that a given fungal taxon or group of fungi plays in the social life of a group of people or a community. Using pictures or fresh sporocarps of edible fungi, the edible mushrooms' cultural significance for a fungal species corresponds with the sum of the scores for "edibility status" given by all informants, divided by the total number of informants. Edibility status scores or frequencies of mention (FM) were assigned through informants' answers to the following question: Do you eat this mushroom? (yes $=1$, no $=0$ ). A Kruskal-Wallis analysis was used 
to test the role of ethnicity on the edibility status score, and how this changes according to the different trophic groups.

\section{Results}

\section{Checklist and diversity of wild edible fungi}

Table 1 presents the checklist of edible fungi recorded from the rainforests of Tshopo. Based on the information found in the literature and ethnomycological inquiries, 78 species of macrofungi are edible.

\section{Trophic groups and distribution of edible fungi}

Edible species belong to 3 trophic groups (saprotrophic, ectomycorrhizal, and termite associated). The saprotrophic taxa (wood and litter decaying fungi) represent the most species-rich group (44 species), followed by the EcM fungi (31 species) and termite-associated taxa (3 species, all Termitomyces). Whereas the number of edible fungi significantly differs between trophic groups, strong variation in the composition of edible fungi is observed between forest types (Fig. 2). Figure 2 shows that the mixed forests, on average, hold the highest diversity in saprotrophic edible fungi, while EcM fungi reach their highest species numbers in the monodominant forests. Edible termite-associated fungi (Termitomyces sp.) show the lowest species numbers and seem equally represented in both mixed and monodominant forests. Considering all fungal trophic groups, plots from monodominant and mixed forests showed similar average numbers of edible species, but variations are more important in the monodominant forests. The result is that the highest numbers of edible species were encountered in some of the monodominant forests, especially in the forest dominated by Gilbertiodendron dewevrei. Within monodominant forests, we observed no significant difference in the number of saprotrophic and EcM fungi (Fig. 3). In contrast, within mixed forests, the number of saprotrophic taxa was significantly higher than in the EcM forests.

\section{Usage of edible fungi}

Almost a third ( 25 species) of the 78 edible species are not used for food by any of the interviewed people (Table 2). This is the case for Cantharellus conspicuus, C. incarnatus, Cookeina tricholoma, Coprinus plicatilis, Dacryopinax spathularia, Gerronema hungo, Hygrocybe coccinea, Hypholoma subviride, Lactifluus gymnocarpus, L. heimii, L. pelliculatus, Lepista sordida, Leucocoprinus discoideus, Macrolepiota dolichaula, Marasmiellus inoderma, Neonothopanus hygrophanus, Paxillus brunneotomentosus, Polyporus arcularius, Psathyrella candolleana, Russula roseostriata, $R$. sese, Tremella fuciformis, Tylopilus balloui, Volvariella parvispora, and Volvopluteus gloiocephalus.

Significant differences were observed in the mean number of ectomycorrhizal and saprotrophic fungi eaten by informants from different ethnic groups (Table 3). Because of their low numbers (three species), differences in the mean numbers of consumed termiteassociated species are evidently small. In general, the most commonly eaten mushrooms (with the highest edibility index) are saprotrophic or woody-decaying fungi (Table 3), the most appreciated ones being Auricularia spp., Marasmius buzungolo, Lentinus squarrosulus, and Schizophyllum commune (Fig. 4). Only a small fraction of EcM fungi, especially from the genus Cantharellus, are reported as a delicacy by some populations. The scores of EcM fungi are even more lowered, knowing that some local populations (Kumu) systematically reject all edible Russula because of their vivid colors. In terms of diversity and quantity, local markets (Yangambi) offer far more saprotrophic than EcM fungi (De Kesel pers. obs.).

\section{Discussion}

This study revealed that wild edible fungi from rainforests of Tshopo belong to several trophic groups. They can be either saprotrophic (growing on dead fallen trunks and litter), or termite associated (growing in mutualistic relation with termites), or ectomycorrhizal, i.e., living in a mutualistic relation with the roots of some vascular plants. Within rainforests from the Yangambi Biosphere reserve and Yoko reserve, relatively high numbers of edible fungi are available. A more quantitative approach, similar to the one used by Yorou et al. [41] or De Kesel et al. [4], is needed to ascertain this, but it seems that the EcM fraction of edible fungi is still underexploited. Although a wide array of edible EcM fungi is available in the primary ectomycorrhizal forests, the interest of local people mainly goes to saprotrophic fungi from the degraded mixed forests. Milenge et al. [39] reported that most of these saprotrophic edible taxa typically grow on wood $(\operatorname{logs})$.

Among the reported edible EcM fungi, only species of the genus Cantharellus are considered of some importance in local consumption [39]. As stated by EyiNdong et al. [18], species of the genus Cantharellus are the most important edible EcM fungi found within rainforests from the central African Congo basin. Most species of Cantharellus are known worldwide as good or even excellent edibles. With its 42 African endemic species, the genus is by far best represented on the African continent [9]. In contrast to the mushroom diet chosen by people from Tshopo, Cantharellus species are massively consumed in the Zambezian region $[4,5,9,10,13,14,42]$.

Although other EcM genera, i.e., Amanita, Lactarius, Lactifluus, and Russula, are common in the forests dominated by Gilbertiodendron dewevrei, these edible EcM fungi do not seem to be at all harvested and consumed by 
Table 1 List of edible fungi from rainforests of Tshopo

\begin{tabular}{|c|c|c|c|c|c|c|}
\hline Species & $\begin{array}{l}\text { Edibility } \\
\text { status }\end{array}$ & $\begin{array}{l}\text { Trophic } \\
\text { group }\end{array}$ & FM & $\begin{array}{l}\text { EMCS } \\
\text { score }\end{array}$ & Supporting literature & $\begin{array}{l}\text { Voucher } \\
\text { specimens }\end{array}$ \\
\hline 1. Agaricus bambusicola Heinem. & Food & Sapr & 24 & 0.2 & Milenge et al. [39] & ADK5267 \\
\hline 2. Agaricus crocopeplus Berk. \& Broome & Food & Sapr & 27 & 0.2 & Milenge et al. [39] & $\begin{array}{l}\text { ADK5747, } \\
\text { MKH073 }\end{array}$ \\
\hline 3. Amanita annulatovaginata Beeli & Food & ECM & 41 & 0.3 & Milenge et al. [39] & MKH016 \\
\hline 4. Amanita echinulata Beeli & Food & EcM & 35 & 0.2 & Milenge et al. [39] & $\begin{array}{l}\text { ADK5938, } \\
\text { MKH159 }\end{array}$ \\
\hline 5. Amanita pudica (Beeli) Walleyn & Food & ECM & 49 & 0.3 & $\begin{array}{l}\text { Degreef et al. [7], De Kesel et al. [4], } \\
\text { Milenge et al. [39] }\end{array}$ & ADK5924 \\
\hline 6. Amanita robusta Beeli & Food & ECM & 49 & 0.3 & Boa [1], Milenge et al. [39] & ADK5236 \\
\hline 7. Armillaria heimii Pegler & Food & Sapr & 78 & 0.5 & $\begin{array}{l}\text { Eyi-Ndong et al. [18], Degreef et al. [7], } \\
\text { Milenge et al. [39] }\end{array}$ & $\begin{array}{l}\text { ADK5230, } \\
\text { MKH046 }\end{array}$ \\
\hline 8. Auricularia cornea Ehrenb. & Food & Sapr & 144 & 0.9 & $\begin{array}{l}\text { De Kesel et al. [16], Boa [1], Eyi-Ndong et al. [18], } \\
\text { Degreef et al. [7], De Kesel et al. [4], Milenge et al. [39] }\end{array}$ & ADK5175 \\
\hline 9. Auricularia delicata (Mont.ex Fr.) Henn. & Food & Sapr & 142 & 0.9 & $\begin{array}{l}\text { De Kesel et al. [16], Boa [1], Degreef et al. [7], } \\
\text { De Kesel et al. [4], Milenge et al. [39] }\end{array}$ & $\begin{array}{l}\text { ADK5169, } \\
\text { MKH214 }\end{array}$ \\
\hline $\begin{array}{l}\text { 10. Calyptella longipes (Cooke \& Massee) W.B. } \\
\text { Cooke }\end{array}$ & Food & Sapr & 12 & 0.1 & Milenge et al. [39] & ADK5868 \\
\hline 11. Cantharellus congolensis Beeli & Food & EcM & 89 & 0.6 & $\begin{array}{l}\text { De Kesel et al. [16], Boa [1], Eyi-Ndong et al. [18], } \\
\text { Degreef et al. [7], De Kesel et al. [4], Milenge et al. [39] }\end{array}$ & $\begin{array}{l}\text { ADK5199, } \\
\text { MKH180 }\end{array}$ \\
\hline $\begin{array}{l}\text { 12. Cantharellus conspicuus Eyssartier, Buyck \& } \\
\text { Verbeken }\end{array}$ & Edible & EcM & 0 & 0.0 & Eyssartier et al. [40] & ADK5937 \\
\hline 13. Cantharellus densifolius Heinem. & Food & EcM & 53 & 0.3 & $\begin{array}{l}\text { Boa [1], Eyi-Ndong et al. [18], Degreef et al. [7], } \\
\text { De Kesel et al. [4], Milenge et al. [39] }\end{array}$ & MKH017 \\
\hline 14. Cantharellus floridulus Heinem. & Food & ECM & 54 & 0.3 & $\begin{array}{l}\text { De Kesel et al. [16], Boa [1], Eyi-Ndong et al. [18], } \\
\text { De Kesel et al. [4], Milenge et al. [39] }\end{array}$ & ADK5670 \\
\hline 15. Cantharellus incarnatus (Beeli) Heinem. & Edible & ECM & 0 & 0.0 & Boa [1], Eyi-Ndong et al. [18] & $\begin{array}{l}\text { MKH069, } \\
\text { MKH166 }\end{array}$ \\
\hline 16. Cantharellus longisporus Heinem. & Food & ECM & 132 & 0.8 & Boa [1], Milenge et al. [39] & $\begin{array}{l}\text { ADK5684, } \\
\text { MKH141 }\end{array}$ \\
\hline 17. Cantharellus luteopunctatus (Beeli) Heinem. & Food & ECM & 51 & 0.3 & Boa [1], Eyi-Ndong et al. [18], Milenge et al. [39] & $\begin{array}{l}\text { ADK5836, } \\
\text { MKH078 }\end{array}$ \\
\hline 18. Cantharellus miniatescens Heinem. & Food & ECM & 39 & 0.2 & [1], Eyi-Ndong et al. [18], Milenge et al. [39] & $\begin{array}{l}\text { ADK5216, } \\
\text { MKH142 }\end{array}$ \\
\hline 19. Cantharellus aff. ruber Heinem. & Food & EcM & 29 & 0.2 & $\begin{array}{l}\text { Boa [1], Degreef et al. [7], De Kesel et al. [4], } \\
\text { Milenge et al. [39] }\end{array}$ & ADK5749 \\
\hline 20. Cantharellus rufopunctatus (Beeli) Heinem. & Food & EcM & 84 & 0.5 & $\begin{array}{l}\text { Boa [1], Eyi-Ndong et al. [18], De Kesel et al. [4], } \\
\text { Milenge et al. [39] }\end{array}$ & $\begin{array}{l}\text { ADK5892, } \\
\text { MKH051 }\end{array}$ \\
\hline 21. Cookeina speciosa (Fr.) Dennis & Food & Sapr & 11 & 0.1 & Eyi-Ndong et al. [18], Milenge et al. [39] & MKH054 \\
\hline 22. Cookeina tricholoma (Mont.) Kuntze & Edible & Sapr & 0 & 0.0 & Boa [1] & ADK5701 \\
\hline 23. Coprinellus disseminatus (Pers.) J.E. Lange & Food & Sapr & 18 & 0.1 & Milenge et al. [39] & ADK5615 \\
\hline 24. Coprinus plicatilis (Curtis) Fr. & Edible & Sapr & 0 & 0.0 & {$[1]$} & - \\
\hline 25. Cotylidia aurantiaca (Pat.) A. L. Welden & Food & Sapr & 14 & 0.1 & Boa [1], Degreef et al. [7], Milenge et al. [39] & $\begin{array}{l}\text { ADK5625, } \\
\text { MKH058 }\end{array}$ \\
\hline $\begin{array}{l}\text { 26. Dacryopinax spathularia (Schwein.) G.W. } \\
\text { Martin }\end{array}$ & Edible & Sapr & 0 & 0.0 & Boa [1], De Kesel et al. [4] & ADK5578 \\
\hline 27. Favolus tenuiculus (P. Beauv.) Fr. & Food & Sapr & 8 & 0.1 & $\begin{array}{l}\text { Eyi-Ndong et al. [18], De Kesel et al. [4], } \\
\text { Milenge et al. [39] }\end{array}$ & $\begin{array}{l}\text { MKH109, } \\
\text { MKH195 }\end{array}$ \\
\hline 28. Gerronema hungo (Henn.) Degreef \& Eyi & Edible & Sapr & 0 & 0.0 & Eyi-Ndong et al. [18] & $\begin{array}{l}\text { MKH057, } \\
\text { MKH201 }\end{array}$ \\
\hline 29. Gymnopilus zenkeri (Henn.) Singer & Food & Sapr & 83 & 0.5 & $\begin{array}{l}\text { Eyi-Ndong et al. [18], De Kesel et al. [4], } \\
\text { Milenge et al. [39] }\end{array}$ & ADK5619 \\
\hline
\end{tabular}


Table 1 List of edible fungi from rainforests of Tshopo (Continued)

\begin{tabular}{|c|c|c|c|c|c|c|}
\hline Species & $\begin{array}{l}\text { Edibility } \\
\text { status }\end{array}$ & $\begin{array}{l}\text { Trophic } \\
\text { group }\end{array}$ & FM & $\begin{array}{l}\text { EMCS } \\
\text { score }\end{array}$ & Supporting literature & $\begin{array}{l}\text { Voucher } \\
\text { specimens }\end{array}$ \\
\hline $\begin{array}{l}\text { 30. Hygrocybe cantharellus (Schwein.) } \\
\text { Murrill }\end{array}$ & Food & Sapr & 28 & 0.2 & Boa [1], Milenge et al. [39] & $\begin{array}{l}\text { ADK5849, } \\
\text { MKH229 }\end{array}$ \\
\hline $\begin{array}{l}\text { 31. Hygrocybe coccinea (Schaeff.) P. } \\
\text { Kummer }\end{array}$ & Edible & Sapr & 0 & 0.0 & Boa [1] & ADK5864 \\
\hline $\begin{array}{l}\text { 32. Hymenagaricus luteolosporus } \\
\text { Heinem.\& Little Flower }\end{array}$ & Food & Sapr & 18 & 0.1 & Milenge et al. [39] & ADK5222 \\
\hline $\begin{array}{l}\text { 33. Hypholoma subviride Berk. } \\
\text { \& M.A. Curtis }\end{array}$ & Edible & Sapr & 0 & 0.0 & Degreef et al. [7] & ADK5891 \\
\hline 34. Lactarius acutus Heim & Food & EcM & 49 & 0.3 & Eyi-Ndong et al. [18], Milenge et al. [39] & $\begin{array}{l}\text { ADK5251, } \\
\text { MKH001 }\end{array}$ \\
\hline $\begin{array}{l}\text { 35. Lactifluus annulatoangustifolius } \\
\text { (Beeli) Buyck }\end{array}$ & Food & EcM & 28 & 0.2 & Boa [1], Milenge et al. [39] & ADK5862 \\
\hline $\begin{array}{l}\text { 36. Lactifluus gymnocarpus } \\
\text { (R. Heim ex Singer) Verbeken }\end{array}$ & Edible & EcM & 0 & 0.0 & Boa [1], Eyi-Ndong et al. [18], De Kesel et al. [4] & MKH135 \\
\hline 37. Lactifluus heimii (Verbeken) Verbeken & Edible & EcM & 0 & 0.0 & Boa [1], De Kesel et al. [4] & MKH170 \\
\hline 38. Lactifluus pelliculatus (Beeli) Buyck & Edible & EcM & 0 & 0.0 & Eyi-Ndong et al. [18], Boa [1] & MKH012 \\
\hline 39. Lentinus squarrosulus Mont. & Food & Sapr & 152 & 1.0 & $\begin{array}{l}\text { De Kesel et al. [16], Boa [1], Eyi-Ndong et al. [18], } \\
\text { De Kesel et al. [4], Milenge et al. [39] }\end{array}$ & $\begin{array}{l}\text { ADK5226, } \\
\text { MKH079 }\end{array}$ \\
\hline 40. Lepista rhodotoides Singer & Food & Sapr & 54 & 0.3 & Milenge et al. [39] & MKH085 \\
\hline 41. Lepista sordida (Schumach.) Singer & Edible & Sapr & 0 & 0.0 & Boa [1], Degreef et al. [7], De Kesel et al. [4] & ADK5254 \\
\hline 42. Leucocoprinus cepaestipes (Sowerby) Pat & Food & Sapr & 19 & 0.1 & Boa [1], Milenge et al. [39] & ADK5206 \\
\hline 43. Leucocoprinus discoideus (Beeli) Heinem & Edible & Sapr & 0 & 0.0 & Boa [1] & ADK5606 \\
\hline $\begin{array}{l}\text { 44. Macrolepiota dolichaula (Berk. \& Broome) } \\
\text { Pegler \& R.W. Rayner }\end{array}$ & Edible & Sapr & 0 & 0.0 & $\begin{array}{l}\text { Boa [1], Eyi-Ndong et al. [18], Degreef et al. [7], } \\
\text { De Kesel et al. [4] }\end{array}$ & $\begin{array}{l}\text { ADK5207, } \\
\text { MKH223 }\end{array}$ \\
\hline 45. Marasmiellus inoderma (Berk.) Singer & Edible & Sapr & 0 & 0.0 & Eyi-Ndong et al. [18], Degreef et al. [7] & $\begin{array}{l}\text { ADK5737, } \\
\text { MKH094 }\end{array}$ \\
\hline 46. Marasmius arborescens (Henn.) Beeli & Food & Sapr & 99 & 0.6 & $\begin{array}{l}\text { Boa [1], Eyi-Ndong et al. [18], [7], De Kesel et al. [4], } \\
\text { Milenge et al. [39] }\end{array}$ & $\begin{array}{l}\text { ADK5255, } \\
\text { MKH231 }\end{array}$ \\
\hline 47. Marasmius bekolacongoli Beeli & Food & Sapr & 62 & 0.4 & $\begin{array}{l}\text { Eyi-Ndong et al. [18], Degreef et al. [7], } \\
\text { De Kesel et al. [4], Milenge et al. [39] }\end{array}$ & $\begin{array}{l}\text { ADK5779, } \\
\text { MKH035 }\end{array}$ \\
\hline 48. Marasmius buzungolo Singer & Food & Sapr & 138 & 0.9 & Boa [1], Eyi-Ndong et al. [18], Milenge et al. [39] & $\begin{array}{l}\text { ADK5760, } \\
\text { MKH121 }\end{array}$ \\
\hline 49. Marasmius confertus Berk. \& Broome & Food & Sapr & 51 & 0.3 & Milenge et al. [39] & $\begin{array}{l}\text { ADK5276, } \\
\text { MKH021 }\end{array}$ \\
\hline $\begin{array}{l}\text { 50. Neonothopanus hygrophanus (Mont.) De } \\
\text { Kesel \& Degreef }\end{array}$ & Edible & Sapr & 0 & 0.0 & Eyi-Ndong et al. [18], De Kesel et al. [4] & $\begin{array}{l}\text { ADK5260, } \\
\text { MKH031 }\end{array}$ \\
\hline $\begin{array}{l}\text { 51. Paxillus brunneotomentosus Heinem. \& } \\
\text { Rammeloo }\end{array}$ & Edible & Sapr & 0 & 0.0 & Degreef et al. [7] & $\begin{array}{l}\text { ADK5655, } \\
\text { MKH129 }\end{array}$ \\
\hline 52. Pleurotus cystidiosus O.K. Mill. & Food & Sapr & 118 & 0.7 & $\begin{array}{l}\text { De Kesel et al. [16], Boa [1], Degreef et al. [7], } \\
\text { Milenge et al. [39] }\end{array}$ & MKH067 \\
\hline 53. Pleurotus flabellatus (Berk. \& Br.) Sacc. & Food & Sapr & 48 & 0.3 & $\begin{array}{l}\text { Eyi-Ndong et al. [18], Degreef et al. [7], } \\
\text { Milenge et al. [39] }\end{array}$ & ADK5771 \\
\hline 54. Pleurotus tuber-regium (Fr.) Fr. & Food & Sapr & 98 & 0.6 & $\begin{array}{l}\text { De Kesel et al. [16], Boa [1], Eyi-Ndong et al. [18], } \\
\text { Degreef et al. [7], De Kesel et al. [4], Milenge et al. [39] }\end{array}$ & MKH106 \\
\hline 55. Polyporus arcularius (Batsch) Fr. & Edible & Sapr & 0 & 0.0 & Boa [1] & MKH107 \\
\hline 56. Psathyrella candolleana (Fr.) Maire & Edible & Sapr & 0 & 0.0 & Boa [1] & ADK5252 \\
\hline $\begin{array}{l}\text { 57. Rubinoboletus luteopurpureus (Beeli) } \\
\text { Heinem. \& Rammeloo }\end{array}$ & Food & EcM & 24 & 0.2 & Boa [1], Milenge et al. [39] & $\begin{array}{l}\text { ADK5192, } \\
\text { MKH235 }\end{array}$ \\
\hline 58. Russula annulata R. Heim & Food & EcM & 32 & 0.2 & Milenge et al. [39] & MKH014 \\
\hline 59. Russula inflata Buyck & Food & EcM & 29 & 0.2 & Milenge et al. [39] & ADK5217 \\
\hline 60. Russula meleagris Buyck & Food & EcM & 35 & 0.2 & Boa [1], Milenge et al. [39] & MKH113 \\
\hline
\end{tabular}


Table 1 List of edible fungi from rainforests of Tshopo (Continued)

\begin{tabular}{|c|c|c|c|c|c|c|}
\hline Species & $\begin{array}{l}\text { Edibility } \\
\text { status }\end{array}$ & $\begin{array}{l}\text { Trophic } \\
\text { group }\end{array}$ & FM & $\begin{array}{l}\text { EMCS } \\
\text { score }\end{array}$ & Supporting literature & $\begin{array}{l}\text { Voucher } \\
\text { specimens }\end{array}$ \\
\hline 61. Russula porphyrocephala Buyck & Food & ECM & 26 & 0.2 & Milenge et al. [39] & $\begin{array}{l}\text { ADK5750, } \\
\text { MKH178 }\end{array}$ \\
\hline 62. Russula pruinata Buyck & Food & EcM & 27 & 0.2 & Milenge et al. [39] & $\begin{array}{l}\text { MKH115, } \\
\text { MKH151 }\end{array}$ \\
\hline 63. Russula roseostriata Buyck & Edible & ECM & 0 & 0.0 & Boa [1], Eyi-Ndong et al. [18] & MKH150 \\
\hline 64. Russula sese Beeli & Edible & EcM & 0 & 0.0 & Boa [1], Eyi-Ndong et al. [18] & MKH114 \\
\hline 65. Russula sesemoindu Beeli & Food & ECM & 49 & 0.3 & Eyi-Ndong et al. [18], Milenge et al. [39] & MKH146 \\
\hline 66. Russula striatoviridis Buyck & Food & ECM & 52 & 0.3 & Boa [1], Eyi-Ndong et al. [18], Milenge et al. [39] & ADK5896 \\
\hline 67. Schizophyllum commune Fr. & Food & Sapr & 160 & 1.0 & $\begin{array}{l}\text { De Kesel et al. [16], Boa [1], Eyi-Ndong et al. [18], } \\
\text { Degreef et al. [7], De Kesel et al. [4], Milenge et al. [39] }\end{array}$ & MKH108 \\
\hline 68. Tapinella panuoides (Fr.) E.-J. Gilbert & Food & Sapr & 26 & 0.2 & Milenge et al. [39] & $\mathrm{MKH043}$ \\
\hline 69. Termitomyces robustus (Beeli) Heim & Food & Term & 128 & 0.8 & $\begin{array}{l}\text { De Kesel et al. [16], Boa [1], Eyi-Ndong et al. [18], } \\
\text { Degreef et al. [7], De Kesel et al. [4], Milenge et al. [39] }\end{array}$ & ADK5242 \\
\hline 70. Termitomyces singidensis Saarim. \& Härk. & Food & Term & 66 & 0.4 & Boa $[1]$ & ADK5852 \\
\hline 71. Termitomyces striatus (Beeli) Heim & Food & Term & 124 & 0.8 & $\begin{array}{l}\text { De Kesel et al. [16], Boa [1], Eyi-Ndong et al. [18], } \\
\text { Degreef et al. [7], De Kesel et al. [4] }\end{array}$ & ADK5179 \\
\hline 72. Tremella fuciformis Berkeley & Edible & Sapr & 0 & 0.0 & Boa $[1]$ & MKH047 \\
\hline $\begin{array}{l}\text { 73. Tricholomopsis aurea (Beeli) Desjardin \& B.A. } \\
\text { Perry. }\end{array}$ & Food & Sapr & 64 & 0.4 & Boa [1], Eyi-Ndong et al. [18] & $\begin{array}{l}\text { ADK5625, } \\
\text { MKH154 }\end{array}$ \\
\hline 74. Trogia infundibuliformis Berk. \& Br. & Food & Sapr & 38 & 0.2 & $\begin{array}{l}\text { Eyi-Ndong et al. [18], De Kesel et al. [4], } \\
\text { Milenge et al. [39] }\end{array}$ & ADK5223 \\
\hline 75. Tylopilus balloui (Peck) Singer & Edible & ECM & 0 & 0.0 & Boa $[1]$ & ADK5950 \\
\hline 76. Volvariella parvispora Heinem. & Edible & Sapr & 0 & 0.0 & Boa [1], De Kesel et al. [4] & ADK5786 \\
\hline 77. Volvopluteus gloiocephalus (DC) Vizzini & Edible & Sapr & 0 & 0.0 & Boa [1] & ADK5641 \\
\hline $\begin{array}{l}\text { 78. Xerocomus spinulosus Heinem. \& Gooss.- } \\
\text { Font. }\end{array}$ & Food & EcM & 12 & 0.1 & De Kesel et al. [4], Milenge et al. [39] & ADK5209 \\
\hline
\end{tabular}

Edible = species known as edible but not reported locally consumed, food = species locally consumed, FM frequency of mention

local populations of the Tshopo province. However, in miombo areas of Burundi $[7,8,43]$, and similar woodlands in the Zambezian $[4,11]$ and Guineo-Soudanian ecozone [15-17], these genera (and Cantharellus) represent a major source of food and income.

Triggering the interest of local people for nontimber forest products requires science-based information about how much of the resource is available, how much and where it can be obtained in a sustainable way, and what its value may be. Based on productivity data of the 50 most common edible species, 1 ha of Katangese miombo woodland produces an average of $148 \mathrm{~kg}$ of fresh fruiting bodies per year [4]. A very similar biomass, i.e., $140.7 \mathrm{~kg} / \mathrm{ha} /$ year, was obtained from 29 edible species monitored in the savanna woodlands of Benin [17]. From miombo woodlands of Katanga in the Democratic Republic of the Congo for instance, $1 \mathrm{~kg}$ of fresh fruiting bodies of Cantharellus spp. costs approximatively 2.8 USD [4]. Local people realize that the yearly revenue obtained from these NTFP goes hand in hand with sustainable use and preservation of host trees and their immediate environment. Losing revenue from not being able to collect EcM fungi is a strong incentive to preserve, or at least make sure, that the forest ecosystem keeps on delivering its services.

In this context, it surely is interesting to monitor natural productions of edible EcM fungi in different types of rainforests of the Democratic Republic of the Congo. So far, this has not been done. Gathering such data is one thing, but convincing local people of changing or widening their diet, i.e., to include more EcM fungi, is another. Several scientists $[7,17,27,44-47]$ have reported the role of culture and tradition on the variety of mushrooms consumed by local people. In the context of the province of Tshopo, taboos resting on mushroom consumption create mycophobia and subsequently decrease the interest for some edible EcM species. In spite of their high nutritional value, most Russula species are not eaten by Kumu people because 


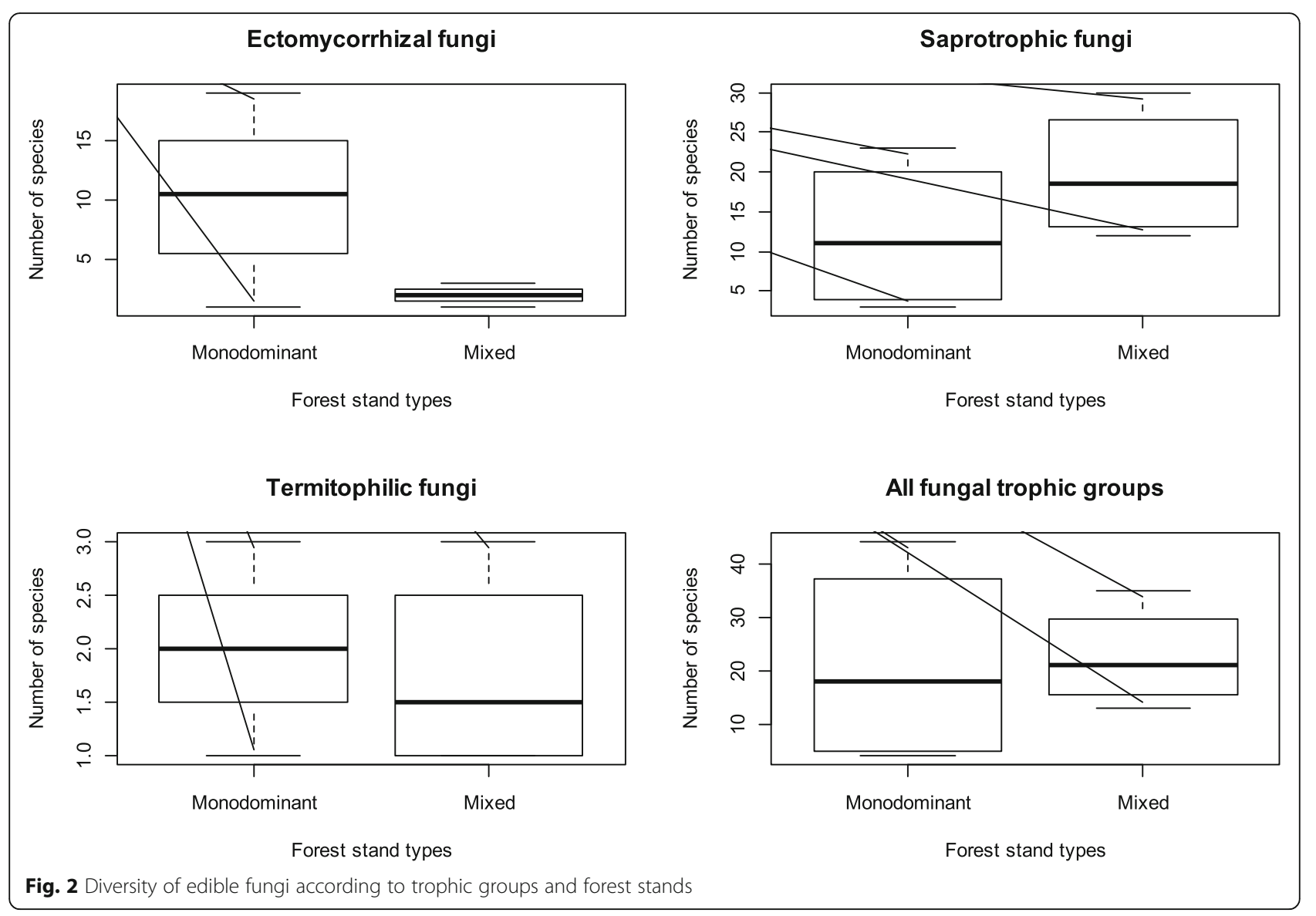

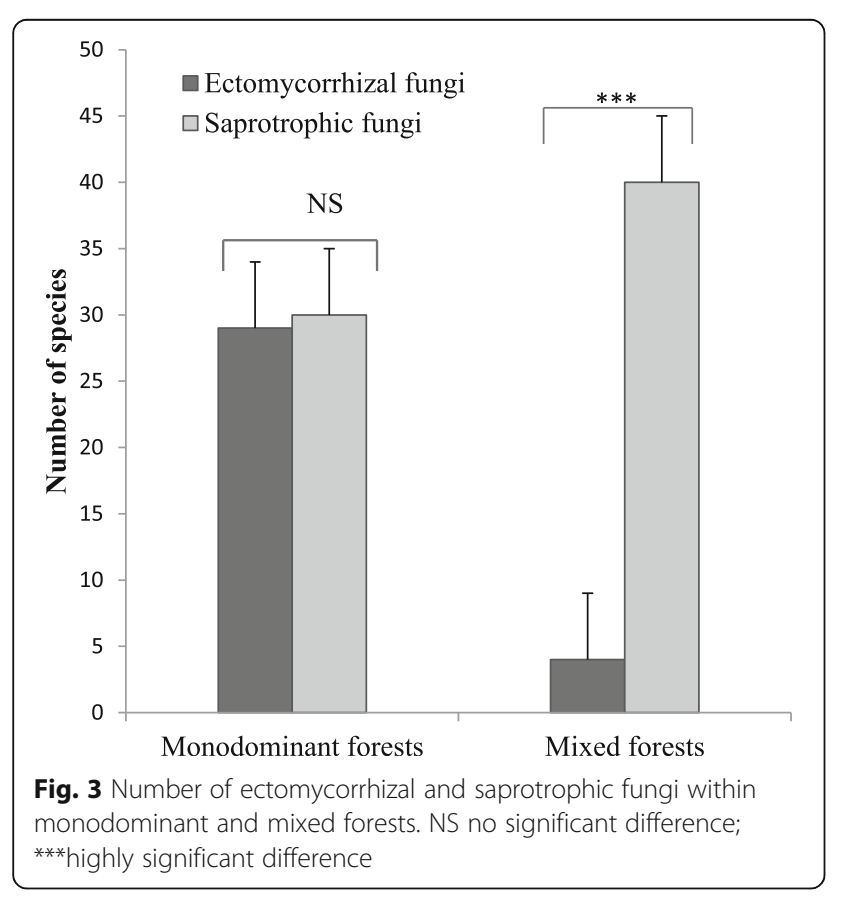

of vivid colors of the cap. Variation in mushroom consumption may also rely on the scarcity of the fruiting bodies [7], and general appreciation is bound to be affected by availability and ease of access to the resource. When some taxa are rare or only available in more remote areas, people may find it difficult to go far to collect them. The result is that mushroom diets can and will shift as a mere result of access and distance to suitable sites [7].

We have no data from eventual hunter-gatherers in the region, but the main reason why people from the Tshopo show a preference for saprotrophic fungi is that these species are found close to the villages and nearby recently cut forests. Because of frequent rains and availability of dead wood, saprotrophic taxa also manage to produce fruit bodies all year round. In contrast, EcM fungi occur abundantly in remote and pristine (or nearly pristine) forests with older growth of Gilbertiodendron dewevrei, Brachystegia laurentii, or Uapaca spp. [19]. With the exception of Cantharellus rufopunctatus, producing fruit bodies at least from April to December [9], most of the edible EcM taxa can only be found during a short period of time. 
Table 2 Edible fungi local names and list of fungi eaten by informants from each ethnic group

\begin{tabular}{|c|c|c|c|c|c|c|c|}
\hline \multirow[t]{2}{*}{ Species } & \multirow[t]{2}{*}{ Local names } & \multicolumn{6}{|c|}{ Ethnic groups } \\
\hline & & Kumu & Lokele & Ngando & Ngelema & Topoke & Turumbu \\
\hline Agaricus bambusicola Heinem. & NA & - & + & + & + & + & + \\
\hline Agaricus crocopeplus Berk. \& Broome & NA & - & + & + & + & + & + \\
\hline Amanita annulatovaginata Beeli & NA & - & + & + & + & + & + \\
\hline Amanita echinulata Beeli & NA & + & + & + & + & + & + \\
\hline Amanita pudica (Beeli) Walleyn & NA & + & + & + & + & + & + \\
\hline Amanita robusta Beeli & Balenda $^{1}$ & + & + & + & + & + & + \\
\hline Armillaria heimii Pegler & Baselekoko $^{3}$ & + & + & + & + & + & + \\
\hline Auricularia cornea Ehrenb. & Batele $e^{1,2,3,4,5,6}$ & + & + & + & + & + & + \\
\hline Auricularia delicata (Mont.ex Fr.) Henn. & Batele $1,2,3,4,5,6$ & + & + & + & + & + & + \\
\hline Calyptella longipes (Cooke \& Massee) W.B. Cooke & Bape-bape $^{6}$ & - & + & + & - & + & + \\
\hline Cantharellus congolensis Beeli & Bolingobopii ${ }^{6}$ & + & + & + & + & + & + \\
\hline Cantharellus conspicuus Eyssartier, Buyck \& Verbeken & NA & - & - & - & - & - & - \\
\hline Cantharellus densifolius Heinem. & NA & + & + & + & + & + & + \\
\hline Cantharellus floridulus Heinem. & Solosolo ${ }^{3}$ & + & + & + & + & + & + \\
\hline Cantharellus incarnatus (Beeli) Heinem. & NA & - & - & - & - & - & - \\
\hline Cantharellus longisporus Heinem. & Baolo-bandei $^{6}$, Abamongo ${ }^{1}$ & + & + & + & + & + & + \\
\hline Cantharellus luteopunctatus (Beeli) Heinem. & Baolo-bandei $^{6}$, Abamongo ${ }^{1}$ & + & + & + & + & + & + \\
\hline Cantharellus miniatescens Heinem. & Lilenda-linenu ${ }^{6}$, Agbalo-gbalo $^{4}$ & + & + & + & + & + & + \\
\hline Cantharellus aff. ruber Heinem. & NA & + & + & + & + & + & + \\
\hline Cantharellus rufopunctatus (Beeli) Heinem. & Bengole-bonyele $^{6}$ & + & + & + & + & + & + \\
\hline Cookeina speciosa (Fr.) Dennis & Batoyi bangwa'a ${ }^{2}$ & - & - & + & - & - & + \\
\hline Cookeina tricholoma (Mont.) Kuntze & Batoyi bangwa'a ${ }^{2}$ & - & - & - & - & - & - \\
\hline Coprinellus disseminatus (Pers.) J.E. Lange & Baku'kulekule ${ }^{6}$ & - & + & + & + & + & + \\
\hline Coprinus plicatilis (Curtis) Fr. & NA & - & - & - & - & - & - \\
\hline Cotylidia aurantiaca (Pat.) A. L. Welden & NA & - & - & + & - & - & + \\
\hline Dacryopinax spathularia (Schwein.) G.W. Martin & NA & - & - & - & - & - & - \\
\hline Favolus tenuiculus (P. Beauv.) Fr. & NA & - & - & + & - & - & + \\
\hline Gerronema hungo (Henn.) Degreef \& Eyi & NA & - & - & - & - & - & - \\
\hline Gymnopilus zenkeri (Henn.) Singer & Bokoo'kina $^{6}$ & + & + & + & + & + & + \\
\hline Hygrocybe cantharellus (Schwein.) Murrill & Adanopila $^{4}$ & - & + & + & + & + & + \\
\hline Hygrocybe coccinea (Schaeff.) P. Kummer & NA & - & - & - & - & - & - \\
\hline Hymenagaricus luteolosporus Heinem.\& Little Flower & Baolo ba'ongolo ${ }^{6}$, Tongwengwe ${ }^{4}$ & - & + & + & + & + & + \\
\hline Hypholoma subviride Berk. \& M.A. Curtis & NA & - & - & - & - & - & - \\
\hline Lactarius acutus Heim & Mongo $^{5}$ & - & + & + & + & + & + \\
\hline Lactifluus annulatoangustifolius (Beeli) Buyck & Belaa'tala ${ }^{3}$ & - & + & + & + & + & + \\
\hline Lactifluus gymnocarpus (R. Heim ex Singer) Verbeken & NA & - & - & - & - & - & - \\
\hline Lactifluus heimii (Verbeken) Verbeken & NA & - & - & - & - & - & - \\
\hline Lactifluus pelliculatus (Beeli) Buyck & Batina lya bakuu ${ }^{6}$ & - & - & - & - & - & - \\
\hline Lentinus squarrosulus Mont. & Bengole $^{6}$ & + & + & + & + & + & + \\
\hline Lepista rhodotoides Singer & Bongolototo $^{6}$, Baselesele ${ }^{2}$ & - & + & + & + & + & + \\
\hline Lepista sordida (Schumach.) Singer & NA & - & - & - & - & - & - \\
\hline Leucocoprinus cepaestipes (Sowerby) Pat & Mbumbuke $^{6}$ & - & + & + & - & + & + \\
\hline Leucocoprinus discoideus (Beeli) Heinem & NA & - & - & - & - & - & - \\
\hline
\end{tabular}


Table 2 Edible fungi local names and list of fungi eaten by informants from each ethnic group (Continued)

\begin{tabular}{|c|c|c|c|c|c|c|c|}
\hline \multirow[t]{2}{*}{ Species } & \multirow[t]{2}{*}{ Local names } & \multicolumn{6}{|c|}{ Ethnic groups } \\
\hline & & Kumu & Lokele & Ngando & Ngelema & Topoke & Turumbu \\
\hline $\begin{array}{l}\text { Macrolepiota dolichaula (Berk. \& Broome) Pegler \& R.W. } \\
\text { Rayner }\end{array}$ & NA & - & - & - & - & - & - \\
\hline Marasmiellus inoderma (Berk.) Singer & NA & - & - & - & - & - & - \\
\hline Marasmius arborescens (Henn.) Beeli & Twendenda $^{5}$, Seeliko ${ }^{3}$ & + & + & + & + & + & + \\
\hline Marasmius bekolacongoli Beeli & Bakoko wa kombe ${ }^{6}$, Toko kombe ${ }^{2}$ & + & + & + & + & + & + \\
\hline Marasmius buzungolo Singer & Mongala ${ }^{6}$, Ndundo $^{3,5}$ & + & + & + & + & + & + \\
\hline Marasmius confertus Berk. \& Broome & Mongala $^{6}$ & + & + & + & + & + & + \\
\hline $\begin{array}{l}\text { Neonothopanus hygrophanus (Mont.) De Kesel \& } \\
\text { Degreef }\end{array}$ & NA & - & - & - & - & - & - \\
\hline Paxillus brunneotomentosus Heinem. \& Rammeloo & NA & - & - & - & - & - & - \\
\hline Pleurotus cystidiosus O.K. Mill. & Bengole $e^{6,2,5}$ & + & + & + & + & + & + \\
\hline Pleurotus flabellatus (Berk. \& Br.) Sacc. & Bengole $e^{6,2,5}$ & + & + & + & + & + & + \\
\hline Pleurotus tuber-regium (Fr.) Fr. & Bengole ya makasi ${ }^{6}$ & + & + & + & + & + & + \\
\hline Polyporus arcularius (Batsch) Fr. & NA & - & - & - & - & - & - \\
\hline Psathyrella candolleana (Fr.) Maire & NA & - & - & - & - & - & - \\
\hline $\begin{array}{l}\text { Rubinoboletus luteopurpureus (Beeli) Heinem. \& } \\
\text { Rammeloo }\end{array}$ & Lofyongi lonyele $^{6}$ & - & + & + & + & + & + \\
\hline Russula annulata R. Heim & Lilianga $^{6}$ & - & + & + & + & + & + \\
\hline Russula inflata Buyck & Balenda ya moindo ${ }^{6}$ & - & + & + & - & + & + \\
\hline Russula meleagris Buyck & Nsalanka'ngaa ${ }^{6}$ & + & + & + & + & + & + \\
\hline Russula porphyrocephala Buyck & Nsololo ${ }^{6}$ & + & + & + & + & + & + \\
\hline Russula pruinata Buyck & Balenda ya kulokoko ${ }^{6}$ & - & + & + & + & + & + \\
\hline Russula roseostriata Buyck & NA & - & - & - & - & - & - \\
\hline Russula sese Beeli & NA & - & - & - & - & - & - \\
\hline Russula sesemoindu Beeli & Malebadja $^{6}$ & - & + & + & + & + & + \\
\hline Russula striatoviridis Buyck & NA & - & + & + & - & + & + \\
\hline Schizophyllum commune Fr. & Bokotoko ${ }^{6}$, Tukumu², Bokokola ${ }^{1,3,4,5}$ & + & + & + & + & + & + \\
\hline Tapinella panuoides (Fr.) E.-J. Gilbert & Tukutuno ${ }^{6}$ & - & + & + & - & + & + \\
\hline Termitomyces robustus (Beeli) Heim & $\begin{array}{l}\text { Limusula }{ }^{6}, \text { Libusula }^{5}, \text { Bululu }^{3} \text {, } \\
\text { Nku'suusu }^{1}\end{array}$ & + & + & + & + & + & + \\
\hline Termitomyces singidensis Saarim. \& Härk. & Limusula $^{6}$, Libusula $^{5}$, Bululu $^{3}$ & - & + & + & - & + & + \\
\hline Termitomyces striatus (Beeli) Heim & Limusula $^{6}$, Libusula ${ }^{5}$, Bululu ${ }^{3}$ & + & + & + & + & + & + \\
\hline Tremella fuciformis Berkeley & NA & - & - & - & - & - & - \\
\hline Tricholomopsis aurea (Beeli) Desjardin \& B.A. Perry. & NA & + & + & + & + & + & + \\
\hline Trogia infundibuliformis Berk. \& Br. & NA & + & + & + & + & + & + \\
\hline Tylopilus balloui (Peck) Singer & NA & - & - & - & - & - & - \\
\hline Volvariella parvispora Heinem. & NA & - & - & - & - & - & - \\
\hline Volvopluteus gloiocephalus (DC) Vizzini & NA & - & - & - & - & - & - \\
\hline Xerocomus spinulosus Heinem. \& Gooss.-Font. & Kwekwele ${ }^{6}$ & - & - & + & - & - & + \\
\hline
\end{tabular}

${ }^{1}$ Kumu, ${ }^{2}$ Lokele, ${ }^{3} \mathrm{Ngando},{ }^{4} \mathrm{Ngelema},{ }^{5}$ Topoke, ${ }^{6}$ Turumbu (mother tongues); " + " indicates edible fungi and " " informants; NA not applicable

\section{Conclusion}

The results from this study reveal that rainforests from the Yangambi Biosphere reserve and Yoko forest reserve of the Tshopo provide a substantial number of edible fungi.
Although not formally measured, there is little doubt that the local populations collect and use only a small fraction of the naturally produced quantity of edible fungi. Most of the locally used taxa are saprotrophic and easily accessed from 
Table 3 Mean numbers of edible fungi eaten by informants from each ethnic group (tribe) \pm standard deviation (SD)

\begin{tabular}{|c|c|c|c|c|c|}
\hline \multirow{2}{*}{$\begin{array}{l}\text { Ethnic } \\
\text { groups }\end{array}$} & \multicolumn{3}{|c|}{ Fungal trophic groups } & \multicolumn{2}{|c|}{ Kruskal-Wallis } \\
\hline & $\begin{array}{l}\text { Ectomycorrhizal } \\
\text { fungi, mean } \pm \\
S D\end{array}$ & $\begin{array}{l}\text { Saprotrophic } \\
\text { fungi, } \\
\text { mean } \pm S D\end{array}$ & $\begin{array}{l}\text { Termitophilic } \\
\text { fungi, } \\
\text { mean } \pm S D\end{array}$ & $p$ value & Significance \\
\hline Turumbu & $8 \pm 3$ & $13 \pm 3$ & $2 \pm 1$ & $<0.001$ & $* * *$ \\
\hline Lokele & $7 \pm 3$ & $12 \pm 3$ & $2 \pm 1$ & $<0.001$ & $* * *$ \\
\hline Topoke & $7 \pm 3$ & $13 \pm 3$ & $2 \pm 1$ & $<0.001$ & $* * *$ \\
\hline Ngando & $19 \pm 3$ & $24 \pm 4$ & $2 \pm 1$ & $<0.001$ & $* * *$ \\
\hline Ngelema & $10 \pm 3$ & $12 \pm 2$ & $2 \pm 1$ & $<0.001$ & $* * *$ \\
\hline Kumu & $8 \pm 2$ & $11 \pm 2$ & $2 \pm 1$ & $<0.001$ & $* * *$ \\
\hline$p$ value & $<0.001$ & $<0.001$ & $>0.05$ & & \\
\hline Significance & $* * *$ & $* * *$ & NS & & \\
\hline
\end{tabular}

The two last columns and lines indicate the $p$ value and significance level of the Kruskal-Wallis test, respectively (NS no significant difference, ${ }^{* * *}$ very high significant difference)

either litter or dead wood. It is the availability, abundance, and proximity of this resource that makes saprotrophic fungi from anthropized environments (secondary forests, clearings, fallow, farmland) most attractive and preferred. As EcM fungi are harder to obtain and less frequent, they are a poorly known and underutilized food source. Whereas in the Zambezian region, the major local interest for edible EcM fungi can be used as a strong incentive for forest conservation; our observations indicate that this is not the case in the Tshopo region.
Some locally considered inedible EcM fungal taxa are in fact perfectly edible and used for food elsewhere in Africa. More research needs to be done, but assessing the natural productivity of edible fungi from the rainforests should help us better understand what these fungi can mean as an ecosystem service to local livelihoods. In combination with the measured interest of local people for this resource, one should be able to develop systems to promote the use of this largely untapped resource.

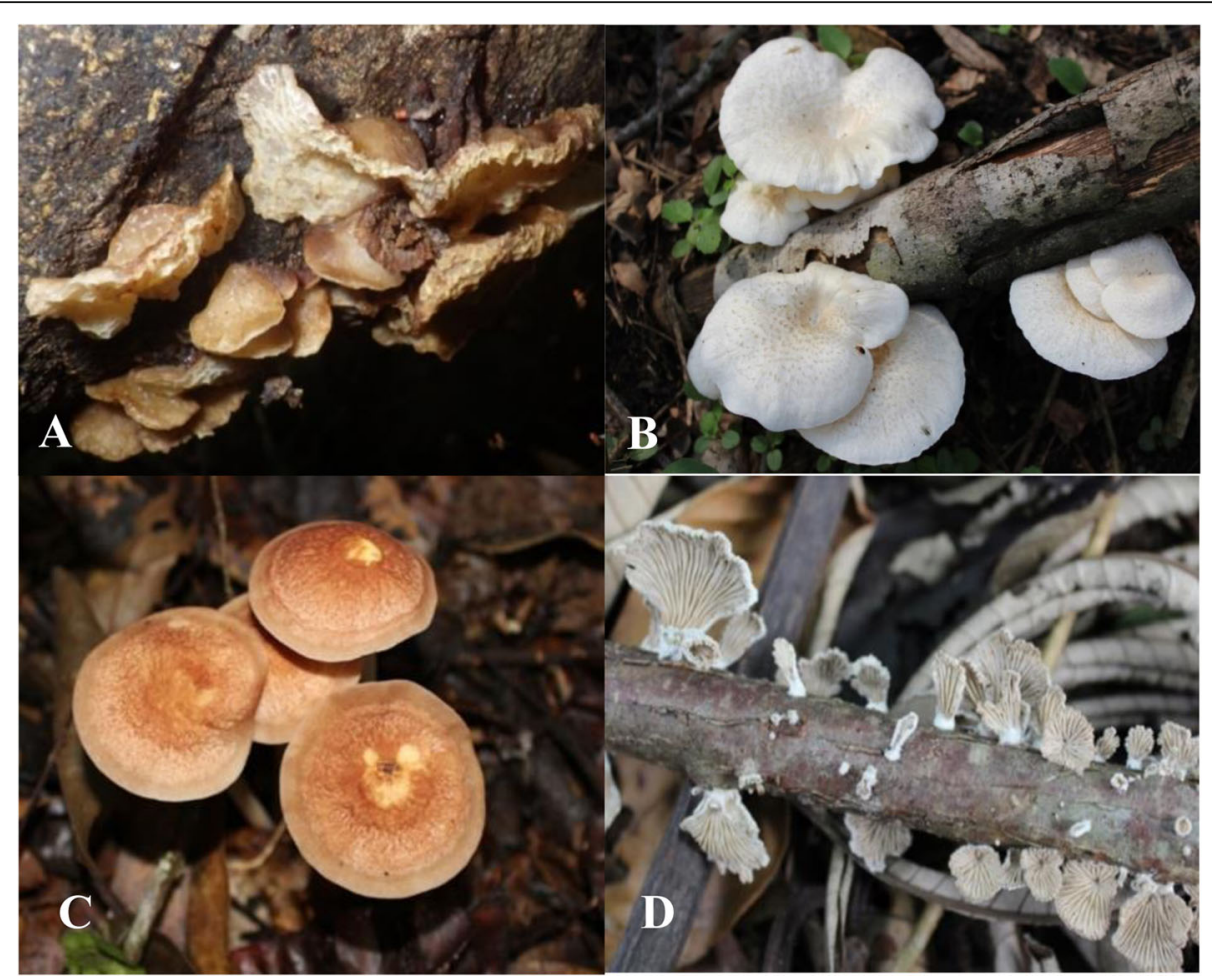

Fig. 4 Four of the most important edible saprotrophic fungi in Tshopo province. a Auricularia delicata. b Lentinus squarrosulus. c Marasmius buzungolo. d Schizophyllum commune 


\section{Abbreviations}

DR Congo: Democratic Republic of the Congo; EcM: Ectomycorrhizal (fungi, forests, trees); EMCS: Edible mushrooms' cultural significance; NTFP: Nontimber forest product; Sapr: Saprotrophic fungi; Term: Termitophilic fungi

\section{Acknowledgements}

The first author acknowledges the PhD scholarship granted by the Center for International Forestry Research (CIFOR) at the University of Kisangani (DR Congo) and the research grant from the International Foundation for Science (IFS/ I-3-D-6072-1) that supported respectively fungal sampling expedition and ethnomycological data collection. Many thanks to the Belgian Global Taxonomy Initiative of the CEBioS program for supporting the taxonomic analysis for species identification at the Meise Botanic Garden in Belgium. A. De Kesel acknowledges the Belgian Federal Science Policy Office (BELSPO) for financing his fieldwork in Yangambi through the project COBIMFO (Congo basin integrated monitoring for forest carbon mitigation and biodiversity). Ratos RISASI, Jules BOMBILE, Antoine MOTOSIA, and Michel MBASI are acknowledged for the assistance and guidance during the sampling expedition and ethnomycological survey campaign.

\section{Authors' contributions}

HMK designed the study; supplied the objectives; collected the data; did the species identification, data analysis, and interpretation; and wrote the first manuscript draft. ADK supplied the tools for identification; did the data collection, species identification (macro- and microscopy), and laboratory work; and is the co-writer and responsible for the corrections to the English text. Both authors read and approved the final manuscript.

\section{Funding}

Center for International Forestry Research (CIFOR), Belgian Federal Science Policy Office (BELSPO), and International Foundation for Science (IFS)

\section{Availability of data and materials}

The data that support the findings of this study have been deposited at the herbarium of the Meise Botanic Garden in Belgium (BR) through the reference codes ADK (for the collection made by André De Kesel) and MKH (for the collection made by Milenge Kamalebo Héritier). Some data are accessible on https://www.efta-online.org/. The overall data are available from the authors.

\section{Ethics approval and consent to participate}

Not applicable

\section{Consent for publication}

Not applicable

\section{Competing interests}

The authors declare that they have no competing interests.

\section{Author details}

${ }^{1}$ Faculté des Sciences, Université de Kisangani, BP 2012, Kisangani, Democratic Republic of the Congo. ${ }^{2}$ Centre de Recherches Universitaires du Kivu (CERUKI-ISP), BP 854, Bukavu, Democratic Republic of the Congo. ${ }^{3}$ Meise Botanic Garden, Nieuwelaan 38, Meise, Belgium.

Received: 7 June 2019 Accepted: 28 January 2020 Published online: 10 February 2020

\section{References}

1. Boa E. Non-wood forest products 17. Wild edible fungi, a global overview of their use and importance to people. Rome: FAO; 2004

2. Chang S-T, Mills PG. Mushrooms: cultivation, nutritional value, medicinal effect and environmental impact: CRC Press; 2004

3. Corrêa RCG, Brugnari T, Bracht A, Peralta RM, Ferreira ICFR. Biotechnological, nutritional and therapeutic uses of Pleurotus spp. (oyster mushroom) related with its chemical composition: a review on the past decade findings. Trends Food Sci Technol. 2016;50:103-17.

4. De Kesel A, Kasongo B, Degreef J. Champignons comestibles du HautKatanga (R D Congo). Abc Taxa 17; 2017.
5. Degreef J, Malaisse F, Rammeloo J, Baudart E. Edible mushrooms of the Zambezian woodland area. Nutritional and ecological approach. Biotechnol Agron Soc Environ. 1997;1(3):221-31.

6. Khatun S, Islam A, Cakilcioglu U, Guler P, Chatterjee NC. Nutritional qualities and antioxidant activity of three edible oyster mushrooms (Pleurotus spp.). NJAS- Wageningen J Life Sci. 2015;72(73):1-5.

7. Degreef J, Demuynck L, Mukandera A, Nyirandayambaje G, Nzigidahera B, De Kesel A. Wild edible mushrooms, a valuable resource for food security and rural development in Burundi and Rwanda. Biotechnol Agron Soc Environ. 2016;20(4):441-52.

8. Buyck B. Flore illustrée des champignons d'Afrique centrale: Russula II (Russulaceae). Bull Jard Bot Nat Belg. 1994a;16:411-542.

9. De Kesel A, Amalfi M, Kasongo B, Yorou NS, Raspé O, Degreef J, Buyck B. New and interesting Cantharellus from tropical Africa. Cryptogam Mycol. 2016;37:283-327. https://doi.org/10.7872/crym/v37.iss3.2016.283.

10. De Kesel A, Malaisse F. Edible wild food: fungi. In: Malaisse F, editor. How to live and survive in Zambezian open forest (Miombo ecoregion). Gembloux: Presses Agronomiques de Gembloux; 2010. p. 41-56.

11. Härkönen M, Niemelä T, Mbindo K, Kotiranta H, Piearce G. Zambian mushrooms and mycology. Norrlinia 29; 2015.

12. Härkönen $\mathrm{M}$, Niemelä T, Mwasumbi L. Tanzanian mushrooms - edible, harmful and other fungi. Norrlinia 10; 2003.

13. Malaisse F. Se nourrir en forêt claire Africaine. Approche écologique et nutritionnelle. Faculté des Sciences agronomiques de Gembloux; 1997.

14. Malaisse F, Guy P. Edible wild vegetable products in the Zambezian woodland area: a nutritional and ecological approach. Ecol Food Nutr. 1985; 18:43-82.

15. Boni S, Yorou NS. Diversité et variabilité inter-ethnique dans la consommation de champignons sauvages de la région de N'Dali au Bénin. Tropicultura. 2015;33(4):266-76.

16. De Kesel A, Codjia JTC, Yorou NS. Guide des champignons comestibles du Bénin. COCO MULTIMEDIA; 2002

17. Yorou SN, De Kesel A. Connaissances ethnomycologiques des peuples Nagot du centre du Bénin (Afrique de l'Ouest). In: Robbrecht E, Degreef J, Friis I, editors. Plant systematics and phytogeography for the understanding of African biodiversity, Proceedings of the XVIth AETFAT Congress 2000 (Meise, National Botanic Garden of Belgium), vol. 71; 2001. p. 627-37. Syst. Geogr. Pl.

18. Eyi-Ndong H, Degreef J, De Kesel A. Champignons comestibles des forêts denses d'Afrique centrale. Taxonomie et identification. Abc Taxa volume 10; 2011.

19. Milenge KH, Nshimba SMH, Masumbuko NC, Nabahungu NL, Degreef J, De Kesel A. Host plants and edaphic factors influence the distribution and diversity of ectomycorrhizal fungal fruiting bodies within rainforests Tshopo, Democratic Republic of the Congo. Afr J Ecol. 2019;00:1-13. https://doi.org/ 10.1111/aje.12595.

20. Lejoly J, Ndjele M-B, Geerinck D. Catalogue-flore des plantes vasculaires des districts de Kisangani et de la Tshopo (R D Congo). Taxonomania. 2010; 4(30):1-308

21. Amani IC. Vegetation patterns and role of edaphic heterogeneity on plant communities in semi-deciduous forests from the Congo basin. PhD thesis, Université Libre de Bruxelles; 2011. p. 198.

22. White F. The vegetation of Africa: Natural resources research, Unesco; 1983.

23. Nshimba S-MH, Etude floristique, écologique et phytosociologique des forêts de l'île Mbiye à Kisangani, R D Congo. PhD thesis, Université Libre de Bruxelles, 2008.

24. Vleminckx J, Drouet T, Amani J, Lisingo J, Lejoly J, Hardy OJ. Impact of fine scale edaphic heterogeneity on tree species assembly in a central African rainforest. J Veg Sci. 2014.

25. Lodge J, Ammirati JF, O'Dell TE, Mueller GM. Collecting and describing macrofungi. In: Mueller GM, Bills G, Foster MS, editors. Biodiversity of fungi: inventory and monitoring methods. San Diego: Elsevier Academic Press; 2004. p. $128-58$

26. Arnolds E. Ecology and coenology of macrofungi in grasslands and moist heathlands in Drenthe, the Netherlands. Part 1. Introduction and synecology. Bibl Mycol. 1981;83:1-410.

27. De Kesel A. A mushrooms dryer for the travelling mycologist. Field Mycol. 2001;2(4):131-3.

28. Heinemann P. Flore iconographique des champignons du Congo: Boletineae. Bull Jard Bot Et, Bruxelles. 1954;3:51-80.

29. Heim R. Flore iconographique des champignons du Congo: Lactarius. Bull Jard Bot Et, Bruxelles. 1955;4:83-97. 
30. Pegler DN. A preliminary agaric flora of East Africa. Kew Bull. 1977;6:615.

31. Heinemann P, Rammeloo J. Flore illustrée des champignons d'Afrique centrale: Gyrodontaceae (Boletineae). Bull Jard Bot Nat Belg. 1983;10:173-98.

32. Heinemann $P$, Rammeloo J. Flore illustrée des champignons d'Afrique centrale: Phylloporus (Boletineae). Bull Jard Bot Nat Belg. 1987;13:277-309.

33. Heinemann P, Rammeloo J. Flore illustrée des champignons d'Afrique centrale: Suillus (Boletaceae, Boletineae), Tubosaeta (Xerocomaceae, Boletinae). Bull Jard Bot Nat Belg. 1989;14:313-335.

34. Buyck B. Flore illustrée des champignons d'Afrique centrale: Russula II (Russulaceae). Bull Jard Bot Nat Belg. 1993;15:337-407.

35. Buyck B. Flore illustrée des champignons d'Afrique centrale: Russula III (Russulaceae). Bull Jard Bot Nat Belg. 1997;17:545-98.

36. Verbeken A, Walleyn R. Fungus flora of tropical Africa. Monograph of Lactarius in tropical Africa. National Botanic Garden (Meise/Belgium), vol. 2; 2010.

37. Gumucio S, Merica M, Luhmann N, Fauvel G, Zompi S, Ronsse, Courcaud A, Bouchon M, Trehin C, Schapman S, Cheminat O, Ranchal H, Simon S. Collecte de données, méthodes quantitatives. L'exemple des enquêtes CAP (connaissance, attitudes et pratiques). Médecins du Monde; 2011.

38. Pieroni A. Evaluation of the cultural significance of wild food botanicals traditionally consumed in northwestern Tuscany, Italy. J Ethnobiol. 2001; 21(1):89-104.

39. Milenge KH, Nshimba SMH, Masumbuko NC, Degreef J, De Kesel A. Uses and importance of wild fungi: traditional knowledge from the Tshopo province in the Democratic Republic of the Congo. J Ethnobiol Ethnomedicine. 2018;14:13. https://doi.org/10.1186/s13002-017-0203-6.

40. Eyssartier G, Buyck B, Verbeken A. Cantharellus conspicuous sp. nov. Cryptogam Mycol. 2002;23(2):95-102.

41. Yorou SN, De Kesel A, Sinsin B, Codjia JTC. Diversité et productivité des champignons comestibles de la forêt classée de Wari-Maro (Bénin, Afrique de l'Ouest). Syst Geogr PI. 2002;71:613-25 http://www.indexfungorum.org/ Names/Names.asp. Accessed 12 Nov 2017.

42. Buyck B. Ubwoba: Les champignons comestibles de l'Ouest du Burundi. AGCD, publication agricole 34; 1994b.

43. Buyck B, Nzigidahera B. Ethnomycological notes from Western Burundi. Belg. J. Bot. 1995;128(2):131-138.

44. Garibay-Orijel R, Caballero J, Estrada-Torres A, Cifuentes J. Understanding cultural significance, the edible mushrooms case. J Ethnobiol Ethnomed. 2007;3:4. https://doi.org/10.1186/1746-4269-3-4.

45. Tibuhwa D. Folk taxonomy and use of mushrooms in communities around Ngorongoro and Serengeti National Park, Tanzania. J Ethnobiol Ethnomedicine. 2012;8:36.

46. Tibuhwa D. Wild mushroom - an underutilized healthy food resource and income generator: experience from Tanzania rural areas. J Ethnobiol Ethnomed. 2013;9:49.

47. Eyi-Ndong HC, Mounguengui S, Attéké C, Obone-Ndong G. Variation of the Consumption of Mushrooms by Pygmies and Bantus in the North of Gabon. Advances in Microbiology. 2014;4:1212-21.

\section{Publisher's Note}

Springer Nature remains neutral with regard to jurisdictional claims in published maps and institutional affiliations.

Ready to submit your research? Choose BMC and benefit from:

- fast, convenient online submission

- thorough peer review by experienced researchers in your field

- rapid publication on acceptance

- support for research data, including large and complex data types

- gold Open Access which fosters wider collaboration and increased citations

- maximum visibility for your research: over $100 \mathrm{M}$ website views per year

At $\mathrm{BMC}$, research is always in progress.

Learn more biomedcentral.com/submissions 